\title{
Simulación 3D Basada en Agentes de Robo y Asalto a Transeúntes
}

\author{
Fabián Beltrán Osnaya, Héctor Rafael Orozco Aguirre, \\ Victor Manuel Landassuri Moreno \\ Universidad Autónoma del Estado de México, Centro Universitario UAEM Valle de México, \\ Atizapán de Zaragoza, Estado de México, México \\ fabian.b.osnaya@gmail.com, hrorozcoa@uaemex.mx,vmlandassurim@uaemex.mx
}

\begin{abstract}
Resumen. Los efectos de la delincuencia son diversos y complejos, desde los traumas psicológicos y físicos que enfrentan las víctimas, a los impactos negativos en la economía e imagen externa de un país. Desde una perspectiva criminológica, la perpetración de un crimen es causada por un conjunto de factores tales como las circunstancias situacionales, contexto social, y los factores a nivel individual. En este artículo, se presenta un nuevo modelo de simulación $3 \mathrm{D}$ en tiempo real de un vecindario apoyado en la criminología científica, para apreciar cómo agentes policías, victimas y delincuentes, se ven inmersos en situaciones de robo y asalto en la vía pública, así como de inspección y arresto.
\end{abstract}

Palabras clave: Agentes, robo, asalto, transeúnte, vía pública, AnyLogic.

\section{D Simulation based on Robbery Agents and Assaults of Passersby}

\begin{abstract}
The effects of crime are diverse and complex, ranging from psychological and physical trauma faced by victims to the negative impacts on the economy and external image of a country. From a criminological perspective, the commission of a crime is caused by a combination of factors such as situational circumstances, social and individual-level factors. In this paper, a new 3D real-time simulation model for a neighborhood is presented, which is supported by scientific criminology, in order to appreciate how police officers, victims and offenders are involved in situations of robbery and assault on public roads as well as inspection and arrest.
\end{abstract}

Keywords: Agents, robbery, assault, passersby, AnyLogic.

\section{Introducción}

En todo el mundo, la acción de caminar o transportarse en la calle o vía pública, implica correr el riesgo de ser asaltado, sobre todo si se vive en las grandes ciudades. 
Las urbes de América Latina son un claro ejemplo de ello. En México, el robo es uno de los principales delitos, el cual es derivado de problemas sociales que siguen a la alza. Según las cifras del secretariado ejecutivo [1], del mes de enero al mes de agosto de 2015 se registraron 998,976 presuntos delitos, de los cuales el Estado de México registra 136,621; el Distrito Federal 111,285 y Baja California Norte 69,614; siendo estas tres entidades federativas las que presentan el mayor número de incidencias reportadas [2].

De acuerdo a la procuraduría general de justicia del estado y a las denuncias presentadas ante agencias del ministerio público, la cifra total de robos registrados es de 372,736 lo que equivale al $37.31 \%$ de los presuntos delitos reportados [3]. De igual manera, se tiene un registro de robo común con una cifra de 365,044 siendo equivalente al $97.93 \%$ con respecto al total de robos registrados. De dicho tipo de robo, resaltan dos de sus categorías: con violencia y sin violencia, ambas a transeúntes, la suma de estas es de 46,296, lo cual es equivalente al $12.68 \%$ del robo común registrado. De los robos a transeúntes, 11,888 son sin violencia y 34,408 con violencia; representando el $25.68 \%$ y el $74.32 \%$ de la suma total de robos, respectivamente [3].

Las cifras obtenidas y mencionadas anteriormente, no indican cuáles son los factores presentes en un robo o asalto en la vía pública, siendo necesario conducir y reforzar investigaciones para obtener orientaciones que permitan emitir probabilidades, causas y efectos de estos problemas sociales.

Este trabajo, está enfocado al área de la criminología científica, mediante él se propone y aplica un nuevo modelo de simulación 3D basada en agentes que pretende dar la pauta de algunas de las causas y probabilidades que originan situaciones de robo y asalto en la vía pública, mediante la definición y generación de comportamientos dinámicos y predefinidos a partir de características dadas a los agentes. En este modelo, se contemplan diversos tipos de robo y asalto a transeúntes. Los robos modelados son el de cartera de hombres y el de bolso de mujeres. En el caso de los asaltos, se tienen de menor a mayor grado de violencia e impacto para la víctima, el asalto simple, el intimidatorio y el agravado.

\section{Estado del arte}

Los criminólogos han demostrado que las características naturales y el diseño de un entorno tienen un impacto significativo en las comunidades y el crimen que en ellas se suscita [4]. La mayoría de los criminales sólo delinquen si están seguros de lograr su cometido, al estar familiarizados con la zona seleccionada y contando con que otros individuos no intervendrán [5]. La situación socioeconómica de un área es particularmente importante, así los robos y asaltos se producen de manera desproporcionada en las zonas con alto nivel socioeconómico, en especial, cuando estas están cerca de áreas con altas tasas de delincuencia [6], los de tipo violento a menudo se asocian con zonas de alto desempleo y un bajo nivel socioeconómico.

En un intento de explicar lo que impulsa a los delincuentes y el efecto que se tiene sobre las víctimas, se han desarrollado diversas teorías criminológicas, entre ellas la 
teoría de las actividades de rutina [7], la cual estipula que por cada crimen que se produce debe estar presente un delincuente motivado y una víctima, además de la ausencia de un guardia capaz de impedirlo. Aunque dicha teoría es de ayuda para establecer lo que los delincuentes, las víctimas y los guardianes hacen en su vida cotidiana, no establece las decisiones de las personas o las acciones que realizan, sin embargo estos factores pueden ser modelados mediante lo que dicen otras teorías.

La teoría del patrón del crimen [8] examina los espacios de actividad que determinarán las áreas que los delincuentes conocen bien para perpetuar un crimen. La teoría de perspectiva de la elección racional [9] sugiere que la decisión de un delincuente puede ser modelada como un proceso formal por el cual los beneficios potenciales de un delito exitoso se comparan contra las pérdidas potenciales si se es aprehendido.

Las ocurrencias del crimen han sido modeladas mediante la técnica estadística de regresión logística, la cual a pesar de haber dado resultados interesantes trabaja en grandes escalas por lo que no toma en cuenta factores locales como: las personas, calles o el entorno de un vecindario. Para hacer frente a este problema se están utilizando simulaciones por computadora las cuales pueden trabajar a escalas mucho más pequeñas, lo cual incluye modelos de interacción espacial y técnicas de micro simulación [10]. Sin embargo, estas técnicas se enfrentan a dificultades con respecto a la incorporación de factores de comportamiento humano. El modelado basado en agentes ofrece la mejor alternativa, ya que estos pueden interactuar entre sí y con su entorno [11]. De esta manera, un agente evalúa individualmente su situación y con base en reglas, toma una decisión sobre qué acción tomar, permitiendo que en él se puedan implementar comportamientos humanos. La tabla siguiente, da un comparativo con los trabajos más similares al aquí presentado, destacando los aportes de éste.

Tabla 1. Comparación con otros modelos de simulación basados en agentes.

\begin{tabular}{|l|l|l|l|l|l|l|}
\hline \multicolumn{1}{|c|}{ Modelo } & Escenario & Agentes & Comportamiento & $\begin{array}{l}\text { Tiempo } \\
\text { real }\end{array}$ & $\begin{array}{c}\text { Plataforma } \\
\text { utilizada }\end{array}$ & \multicolumn{1}{|c|}{ Delitos } \\
\hline $\begin{array}{l}\text { Canessa y } \\
\text { Quezada [12] }\end{array}$ & Simple & $\begin{array}{l}\text { Ladrón } \\
\text { Polića }\end{array}$ & Predefinido & No & Netlogo & Robo simple \\
\hline Devia [13] & Simple & $\begin{array}{l}\text { Ladrón } \\
\text { Policía } \\
\text { Ciudadano }\end{array}$ & Semi-dinámico & No & Repast Simphony & $\begin{array}{l}\text { Robo simple por } \\
\text { sorpresa y con } \\
\text { violencia }\end{array}$ \\
\hline Amrutha [14] & $\begin{array}{l}\text { Muy } \\
\text { básico }\end{array}$ & $\begin{array}{l}\text { Ladrón } \\
\text { Ciudadano } \\
\text { Policía }\end{array}$ & Predefinido & No & Netlogo & $\begin{array}{l}\text { Robo simple en } \\
\text { casa }\end{array}$ \\
\hline $\begin{array}{l}\text { Malleston } \\
\text { [15] }\end{array}$ & Simple & $\begin{array}{l}\text { No } \\
\text { especifica } \\
\text { do }\end{array}$ & Predefinido & No & $\begin{array}{l}\text { Netlogo (á́n no } \\
\text { implementada) }\end{array}$ & Robo simple \\
\hline $\begin{array}{l}\text { El aquí } \\
\text { propuesto }\end{array}$ & Complejo & $\begin{array}{l}\text { Ladrón } \\
\text { Policía } \\
\text { Ciudadano }\end{array}$ & $\begin{array}{l}\text { Predefinido y } \\
\text { dinámico }\end{array}$ & Sí & AnyLogic & $\begin{array}{l}\text { - Asalto simple, } \\
\text { intimidatorio y } \\
\text { agravado } \\
\text { - Robo de cartera } \\
\text { yolso }\end{array}$ \\
\hline
\end{tabular}




\section{Modelo de simulación 3D propuesto}

La Fig. 1 muestra la implementación basada en agentes del modelo propuesto, en la cual se contempla un entorno virtual $3 \mathrm{D}$ que fue modelado y programado en la versión para investigador de la plataforma AnyLogic [16]. Este entorno incluye la representación de un escenario para un vecindario ordinario como cualquier otro.

La plataforma AnyLogic fue elegida debido a que es una herramienta idónea para simulaciones 3D basadas en agentes, ya que permite crear y programar comportamientos en escenarios multiagente, para simular fenómenos y problemas sociales, tal es el caso del robo y asalto a transeúntes, mediante el modelado de sistemas complejos que evolucionan con el tiempo. En estos sistemas, el programador da un comportamiento dinámico mediante instrucciones en diagramas de estado a agentes que trabajan de manera independiente o colectiva. Cabe destacar que esta herramienta permite definir diferentes tipos de agentes a partir de sus características, denotadas por parámetros y variables; así como de sus comportamientos, denotados como funciones, que pueden tener cada uno de ellos. El período de simulación de un entorno virtual en AnyLogic es en tiempo real y se mide en segundos, el cual puede ser acelerado para obtener resultados de manera más rápida, siempre en concordancia con un tiempo equivalente del mundo físico.

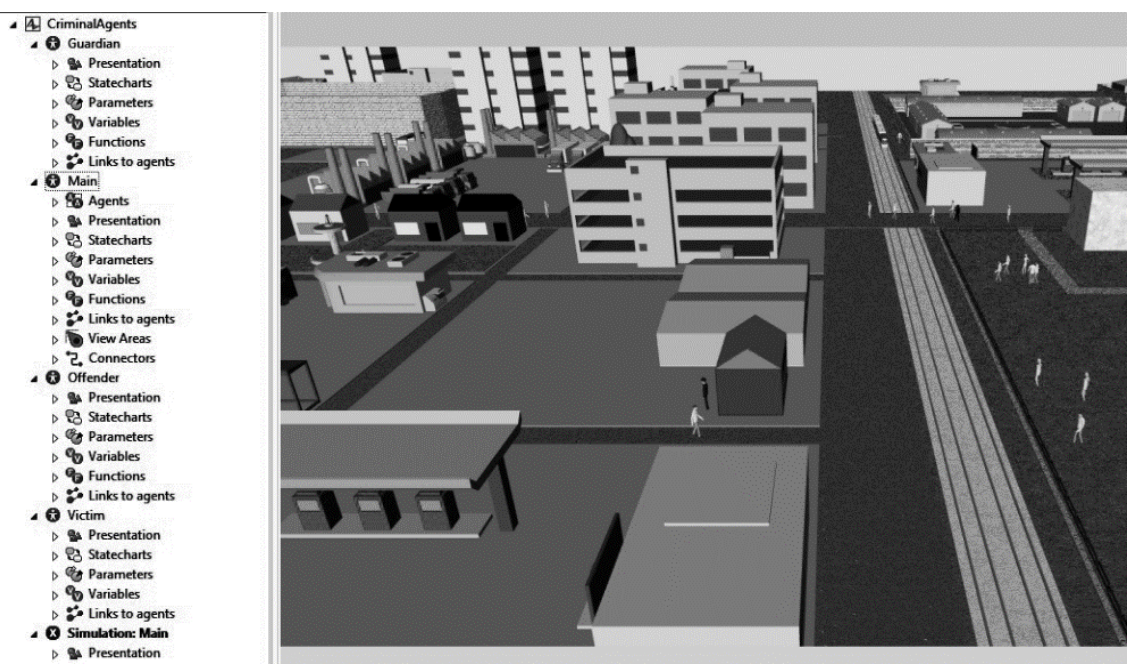

Fig. 1. Creación del modelo de simulación 3D basada en agentes de robo y asalto a transeúntes.

En la vía pública del escenario creado se localizan tres diferentes tipos de agentes, los cuales son descritos como sigue:

- Agentes delincuentes o criminales: quienes siempre están en busca de objetivos (víctimas) que podrían ser fácilmente atacados (ver Fig. 2 y Tabla 2).

- Agentes objetivos o víctimas: cada uno de estos puede ser cualquier agente de la población no delincuente que se encuentra dentro del radio de acción de un agente 
delincuente, con una cierta probabilidad de ser abordado para sufrir un robo o asalto (ver Fig. 3 y Tabla 3).

- Agentes guardianes o policías: están patrullando las calles en busca de agentes sospechosos para ser inspeccionados y posiblemente arrestados (ver Fig. 4 y Tabla 4).

\subsection{Comportamiento de los agentes}

La implementación de los algoritmos para que una situación de robo o asalto pueda ocurrir está basada en los diagramas de estado mostrados en las Fig. 2, Fig. 3 y Fig. 4. Estos algoritmos ofrecen la ventaja de dar la pauta de algunas de las causas y probabilidades que originan situaciones de robo y asalto en la vía pública, con la desventaja de que no se consideran factores sociales y cognitivos en las características de los agentes. Para que ocurra un robo o asalto deben cumplirse los siguientes hechos:

1. Un agente delincuente elige a un agente objetivo: al ocurrir este suceso el primero acelera su paso y empieza a seguir al segundo.

2. El agente delincuente se encuentra cerca del agente objetivo: dado este suceso, el primero está listo para atacar al segundo, pero esto sólo sucede cuando no hay otros agentes testigos potenciales dentro del radio de acción del primero.

3. El ataque se lleva a cabo: el agente delincuente roba o asalta al agente objetivo, el primero puede quedarse junto al segundo por algún tiempo para disfrutar de lo que ha hecho y luego se aleja para buscar un nuevo objetivo potencial. Sin embargo, si un agente delincuente no puede encontrar un buen momento para atacar a un agente objetivo, se da por vencido pasado un tiempo. Por último, la labor de los agentes guardianes es inspeccionar a cualquier agente ciudadano sospechoso, para arrestarlo en caso de que tenga un arma, o bien, se encuentre bajo el abuso de sustancias, de lo contrario, lo deja libre para seguir patrullando la calle.
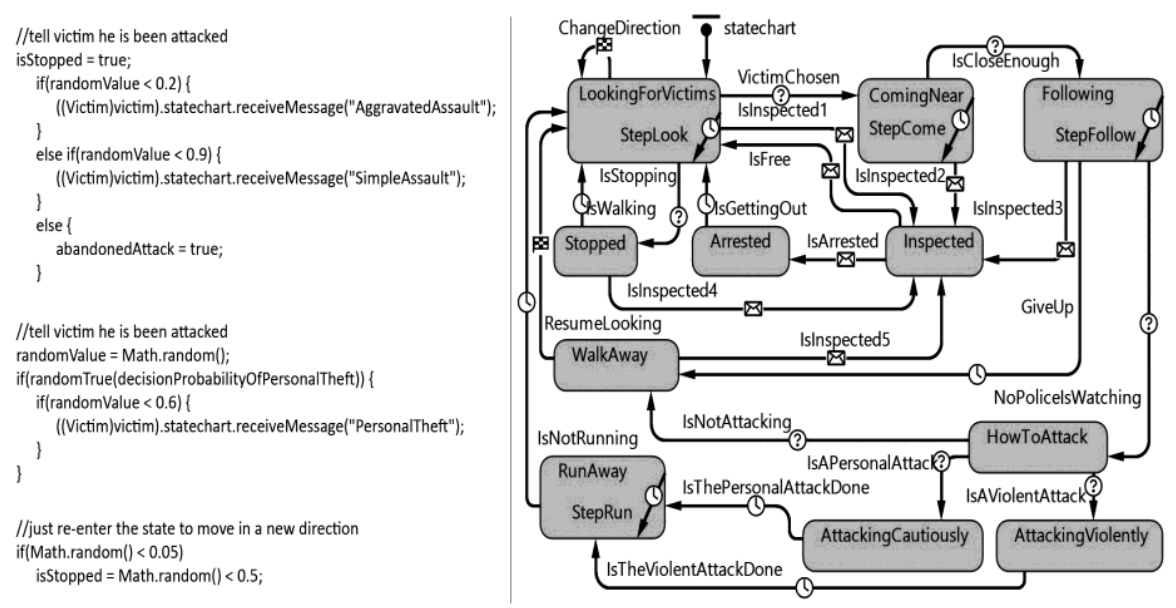

Fig. 2. Fragmentos del comportamiento de un agente delincuente y su diagrama de estados. 
Fabián Beltrán Osnaya, Héctor Rafael Orozco Aguirre, Victor Manuel Landassuri Moreno
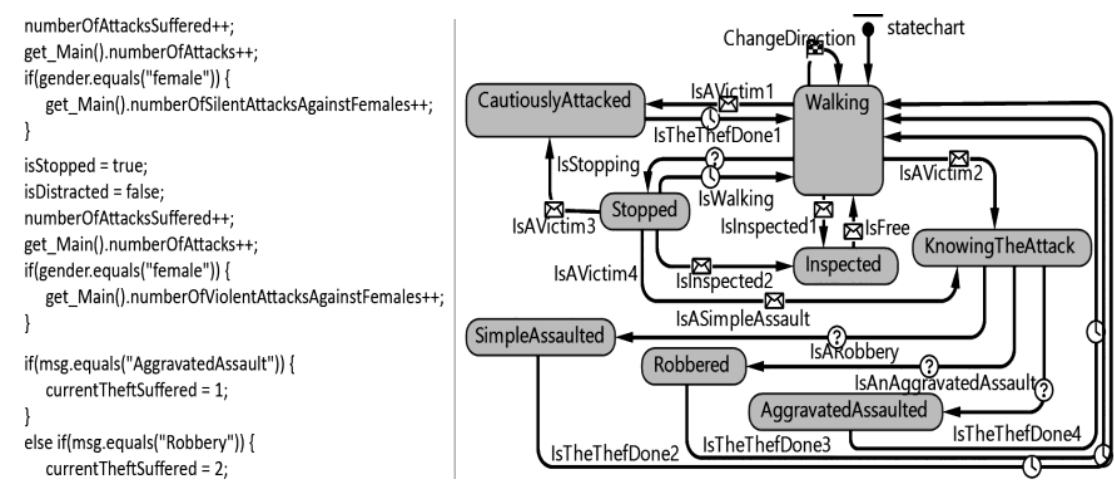

Fig. 3. Fragmentos del comportamiento de un agente objetivo y su diagrama de estados
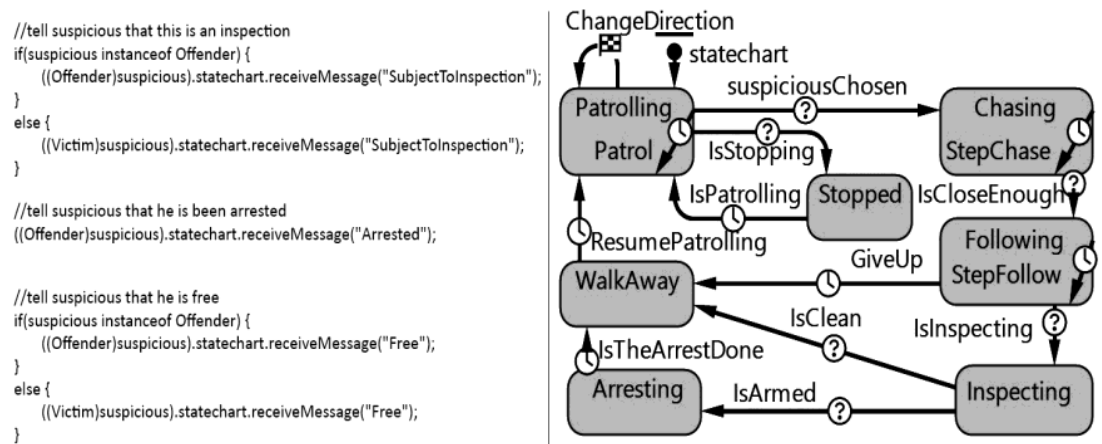

Fig. 4. Fragmentos del comportamiento de un agente guardián y su diagrama de estados.

\subsection{Características de los agentes}

Las principales características de los agentes presentes en el modelo propuesto se plasman en las siguientes tablas:

Tabla 2. Características del agente delincuente.

\begin{tabular}{|l|l|l|}
\hline \multicolumn{1}{|c|}{ Nombre } & \multicolumn{1}{|c|}{ Valores } & \multicolumn{1}{c|}{ Descripción } \\
\hline gender & $\{$ "male", "female" $\}$ & $\begin{array}{l}\text { Representa el género del agente, donde "male" es masculino y } \\
\text { "female" indica femenino. }\end{array}$ \\
\hline typePerson & {$[0,1)$} & $\begin{array}{l}\text { Acorde a una división equitativa del rango de valores y del género, } \\
\text { el agente puede tener una personificación de una enfermera, una } \\
\text { persona ordinaria, un trabajador de la construcción, un empleado de } \\
\text { oficina, un médico, un niño, una mujer rubia, una mujer morena, una } \\
\text { niña. }\end{array}$ \\
\hline hasAWeapon & true, false $\}$ & El agente puede o no portar un arma para cometer un asalto. \\
\hline isUnderSubstanceAbuse & $\{$ true, false $\}$ & $\begin{array}{l}\text { Indica cuando el agente se halla bajo el efecto del abuso de alguna } \\
\text { substancia. }\end{array}$ \\
\hline victim & Agent & $\begin{array}{l}\text { Habla del agente objetivo que ha sido seleccionado para ser } \\
\text { abordado. }\end{array}$ \\
\hline isDistracted & $\{$ true, false $\}$ & $\begin{array}{l}\text { El agente se halla distraído o no lo cual puede aumentar el riesgo de } \\
\text { que sea una víctima a ser abordada. }\end{array}$ \\
\hline isStopped & true, false $\}$ & Estando detenido el agente puede ubicar a una posible víctima. \\
\hline
\end{tabular}


Tabla 3. Características del agente objetivo.

\begin{tabular}{|l|l|l|}
\hline \multicolumn{1}{|c|}{ Nombre } & \multicolumn{1}{|c|}{ Valores } & \multicolumn{1}{c|}{ Descripción } \\
\hline gender & $\{$ "male", "female" $\}$ & $\begin{array}{l}\text { Representa el género del agente, donde "male" es masculino y } \\
\text { "female" indica femenino. }\end{array}$ \\
\hline typePerson & {$[0,1)$} & $\begin{array}{l}\text { Acorde a una división equitativa del rango de valores y del género, } \\
\text { el agente puede tener una personificación de una enfermera, una } \\
\text { persona ordinaria, un trabajador de la construcción, un empleado de } \\
\text { oficina, un médico, un niño, una mujer rubia, una mujer morena, una } \\
\text { niña. }\end{array}$ \\
\hline CurrentTheftSuffered & {$[1,5]$} & $\begin{array}{l}\text { Empleada para asignar el tipo de atraco que acaba de sufrir el agente, } \\
\text { donde 1 es asaltado agravado, 2 es asalto intimidatorio, 3 es asalto } \\
\text { simple, 4 es robo de cartera para un agente masculino y 5 es robo de } \\
\text { bolso para un agente femenino. }\end{array}$ \\
\hline $\begin{array}{l}\text { hasMoreMoneyOrOther } \\
\text { Belongings }\end{array}$ & $\{$ true, false $\}$ & $\begin{array}{l}\text { Indica si el agente posee o no más dinero o pertenencias de las cuales } \\
\text { sufra el riesgo de ser asaltado o robado. }\end{array}$ \\
\hline isDistracted & $\{$ true, false $\}$ & $\begin{array}{l}\text { El agente se halla distraído o no lo cual puede aumentar el riesgo de } \\
\text { que sea una víctima a ser abordada. }\end{array}$ \\
\hline isStopped & $\{$ true, false $\}$ & $\begin{array}{l}\text { Estando detenido el agente se vuelve más vulnerable como posible } \\
\text { víctima de robo o asalto. }\end{array}$ \\
\hline
\end{tabular}

Tabla 4. Características del agente guardián.

\begin{tabular}{|l|l|l|}
\hline \multicolumn{1}{|c|}{ Nombre } & \multicolumn{1}{|c|}{ Valores } & \multicolumn{1}{c|}{ Descripción } \\
\hline gender & $\{$ "male", "female" $\}$ & $\begin{array}{l}\text { Representa el género del agente, donde "male" es masculino y } \\
\text { "female" indica femenino. }\end{array}$ \\
\hline isDistracted & $\{$ true, false $\}$ & $\begin{array}{l}\text { El agente se halla distraído o no lo cual puede aumentar el riesgo de } \\
\text { que exista un robo o asalto. }\end{array}$ \\
\hline isStopped & $\{$ true, false $\}$ & $\begin{array}{l}\text { Estando detenido el agente puede inspeccionar el comportamiento } \\
\text { de otros agentes. }\end{array}$ \\
\hline suspicious & Agent & Corresponde al agente que es identificado como sospechoso \\
\hline
\end{tabular}
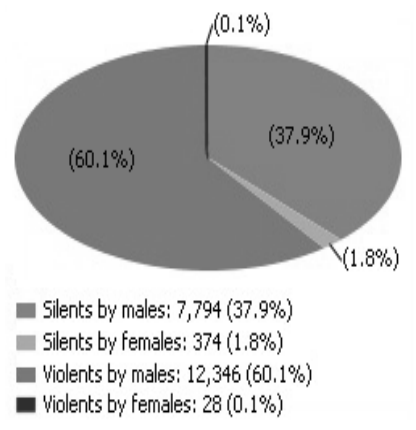

Attempted thefts committed by sex

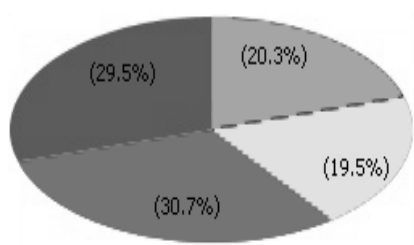

$$
\begin{aligned}
& \square \text { Silents against males: 4,169(20.3\%) } \\
& \square \text { Silents against females: 3,999(19.5\%) } \\
& \square \text { Violents against males: } 6,304(30.7 \%)
\end{aligned}
$$

- Violents against females: $6,070(29.5 \%)$

\section{Attempted thefts suffered by sex}

Fig. 5. Intentos de robo y asalto cometidos (izquierda) y sufridos (derecha) por sexo, sin agentes guardianes.

\subsection{Casos de estudio}

Para validar el modelo propuesto se corrió una simulación a un año para los siguientes casos de estudio:

1. Simulación sin agentes guardianes: en este primer escenario de simulación se contempló una población de agentes dada por: 2000 agentes objetivos, 995 del género femenino y 1005 del género masculino; 50 agentes delincuentes, 10 del 
género femenino y 40 del género masculino, entre los cuales 1 mujer y 14 hombres fueron portadores de un arma, 1 mujer y 11 hombres estuvieron bajo el abuso de sustancias. Los resultados de esta simulación se muestran en la Fig. 5 y la Fig. 6.
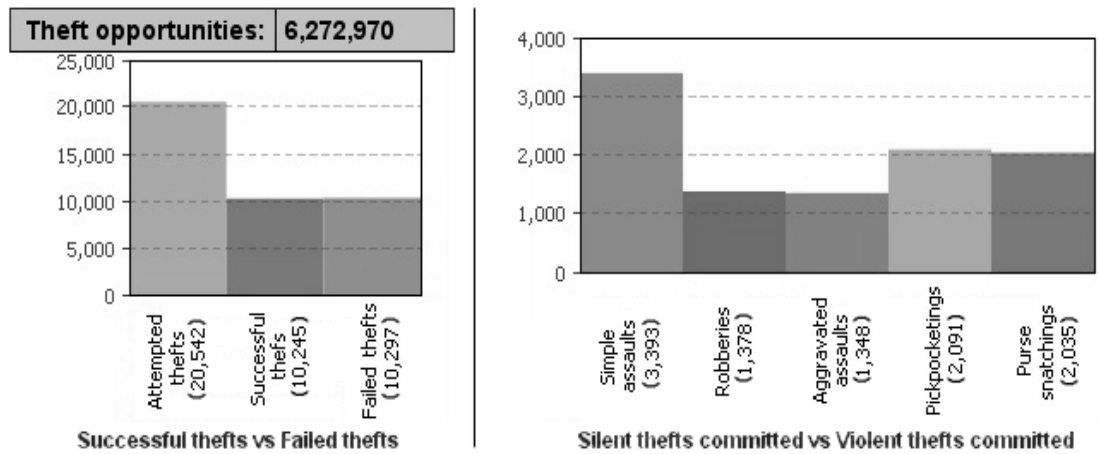

Silent thefts committed vs Violent thefts committed

Fig. 6. Ataques exitosos vs fallidos (izquierda) y aquellos (derecha) que fueron asaltos (violentos) y robos (silenciosos), sin agentes guardianes.

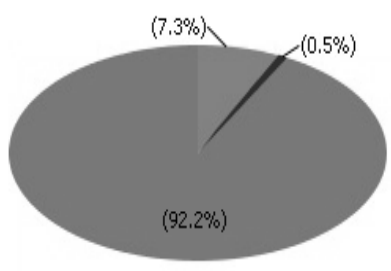

- Silents by males: $1,609(7.3 \%)$

- Silents by females: $117(0.5 \%)$

- Violents by males: $20,464(92.2 \%)$

- Violents by females: $0(0,0 \%)$

Attempted thefts committed by sex

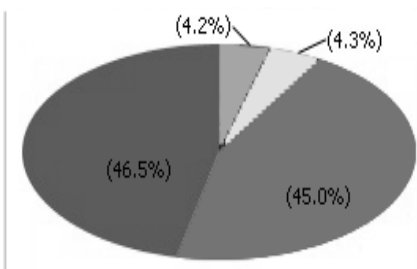

- Silents against males: $445(4.2 \%)$

- Silents against females: $454(4.3 \%)$

- Violents against males: $4,727(45.0 \%)$

- Violents against females: $4,889(46.5 \%)$

Attempted thefts suffered by sex

Fig. 7. Intentos de robo y asaltos cometidos (izquierda) y sufridos (derecha) por sexo, con agentes guardianes.
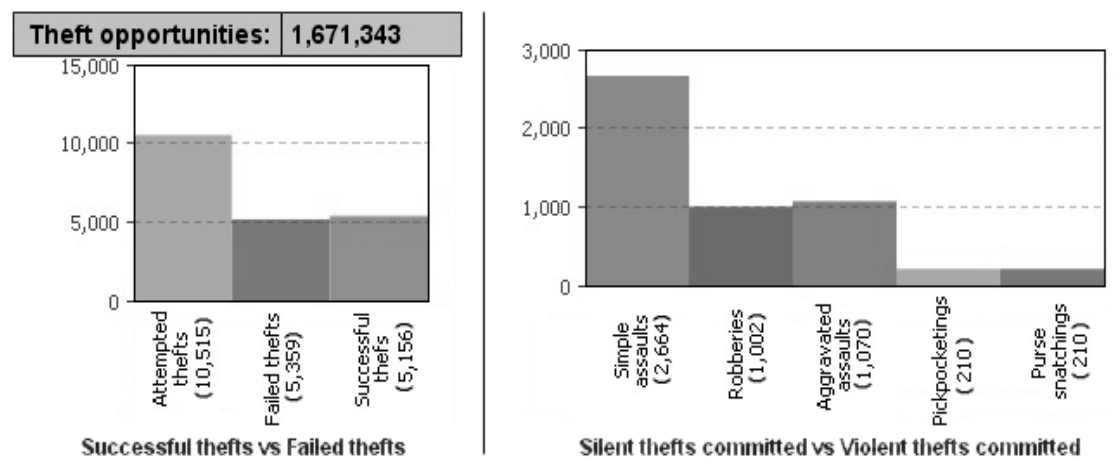

Fig. 8. Ataques exitosos vs fallidos (izquierda) y aquellos (derecha) que fueron asaltos (violentos) y robos (silenciosos), con agentes guardianes. 
2. Simulación con agentes guardianes: para este segundo escenario de simulación se contempló una población de agentes dada por: 2000 agentes objetivos, 991 del género femenino y 1009 del género masculino; 50 agentes delincuentes, 19 del género femenino y 41 del género masculino, entre los cuales 7 hombres fueron portadores de un arma y 10 hombres estuvieron bajo el abuso de sustancias; 50 agentes guardianes, 8 del género femenino y 42 del género masculino, los cuales en total efectuaron 49,305 inspecciones a agentes sospechosos y sólo 164 de ellas fueron arrestos. Para esta simulación, los resultados que se obtuvieron se presentan en la Fig. 7 y la Fig. 8.

En la Fig. 5 y la Fig. 7 los intentos y ataques violentos corresponden a asaltos, mientras que los silenciosos indican robos.

\section{Discusión de resultados}

Cuando existe una población de agentes con igual número de delincuentes que de guardianes (ver Fig. 8), se registraron 1,671,343 oportunidades para intentar cometer ya sea robo o asalto. De esta cifra, se obtiene que sólo el $0.62 \%$ cayeron en intentos realizados, siendo el $49.03 \%$ exitosos y el $50.97 \%$ fallidos. De los crímenes que fueron exitosos, el $91.85 \%$ fue por asalto, teniendo al asalto simple como el de mayor incidencia.

En caso de tener una población de agentes en la cual no se cuenta con la presencia de algún guardián (ver Fig. 6), se observa que la cifra de oportunidades para intentar cometer robo o asalto casi se cuadruplica. Esta cifra fue de 6,272,970, de la cual se tiene que el $0.32 \%$ corresponde a intentos realizados, teniendo el $49.87 \%$ y el $50.13 \%$ como exitosos y fallidos, respectivamente. De la cifra de exitosos, el $59.72 \%$ fue por asalto, teniendo de nueva cuenta al asalto simple como tipo de crimen con mayor incidencia y el $40.28 \%$ por robo, teniendo ligeramente más robos de carteras que de bolsos.

\section{Conclusiones y trabajo futuro}

El modelado y la simulación basados en agentes han sido aplicados en diversas áreas del conocimiento, puesto que ofrecen la ventaja de poder reflejar de manera virtual a nivel individual o colectivo problemas y fenómenos sociales [17]. Sin embargo, en este artículo aquellos que abordan la delincuencia y la criminología fueron los que se tomaron como base para crear el modelo de simulación 3D presentado. Este modelo se distingue del resto en que se propone el uso de diagramas de estado para especificar y modelar comportamientos dinámicos y predefinidos con base en las características de los agentes creados. Estos comportamientos provocan que los agentes víctimas sufran un ataque según las probabilidades y oportunidades aprovechadas por los criminales.

Las principales ventajas que ofrece la implementación del modelo de simulación propuesto en AnyLogic son la recreación de un escenario realista 3D de un vecindario 
en donde se puede observar a nivel individual el comportamiento de cada agente y a nivel estadístico la cantidad de oportunidades presentes durante el periodo de simulación para que ocurra un robo o un asalto y como la presencia de más agentes guardianes contribuye a que estas disminuyan y sea menor la cantidad de aquellos intentos que puedan resultar exitosos. Además, en la simulación se puede apreciar cómo es llevado a cabo el patrullaje para inspeccionar y arrestar, no obstante, aunque son muchas las inspecciones el número de arrestos es mínimo, lo cual dice que la manera de llevar a cabo esta actividad policiaca no ofrece muchas ventajas en la vida real y debe ser replanteada para un mayor rendimiento de los policías en las calles.

Es amplio el número de futuras aplicaciones basadas en la extensión y mejora del modelo propuesto. Estas aplicaciones pueden incluir la consideración de estrategias de predicción, prevención y reducción de los índices de robos y asaltos, por ejemplo al dar la pauta para que en el mundo real se lleven a cabo las prácticas puestas en marcha en los escenarios de simulación virtual.

Finalmente, se tiene contemplado el incluir el uso de las características cognitivas y sociales ligadas a situaciones de robo y asalto en los agentes, para tener un modelo más realista. Al igual, se pueden emplear en complemento con los diagramas de estado el uso de esquemas de inferencia tales como lógica difusa para especificar mediante reglas de inferencia las relaciones y situaciones entre tipos de robos y asaltos que puedan ocurrir. Dichas reglas evaluarían los rangos de pertenencia de todo factor involucrado, como lo son las características y estado de los agentes, así como del entorno simulado para determinar ante situaciones de incertidumbre cuando un robo o asalto es más probable que ocurra, o bien, una inspección se haga cuando en efecto un sospechoso tiene mayor certeza de ser un delincuente y deba ser arrestado. Además, para investigaciones posteriores se extenderá el tipo de crímenes o delitos modelados a otros derivados del fuero común [1], [2], [3].

\section{Referencias}

1. Secretariado Ejecutivo, http://secretariadoejecutivo.gob.mx/index.php

2. Incidencia Delictiva del Fuero Común 2015, http://secretariadoejecutivo.gob.mx/docs/ pdfs/estadisticas\%20del\%20fuero\%20comun/Cieisp2015_082015.pdf

3. Incidencia Delictiva Nacional, http://secretariadoejecutivo.gob.mx/incidenciadelictiva/incidencia-delictiva-acumulado.php

4. Bottoms, A. E., Claytor, A., Wiles, P.: Housing markets and residential community crime careers: A case study from Sheffield. In: Nicholas Fyfe, David Evans, and David Herbert, editors, Crime, Policing and Place - Essays in Environmental Criminology, chapter 7, Routledge (1992)

5. Brown, B. B., Bentley, D. L.: Residential burglars judge risk: The role of territoriality. Journal of Environmental Psychology, vol. 13, pp. 51-61, Elsevier (1993)

6. Baldwin, J. Bottoms, A. E.: The Urban Criminal: A Study in Sheffield. Tavistock Publications, London (1976)

7. Cohen, L. E., Felson, M.: Social Change and Crime Rate Trends: A Routine Activity Approach. American Sociological Review, vol. 44, pp. 588-608, Elsevier (1979)

8. Brantingham, P., Brantingham, P.: Patterns in Crime. The Journal of Criminal Law and Criminology, vol. 76, no. 2, pp. 540-543, New York: Macmillan (1984) 
9. Cornish, D., Clarke, R. V.: The Reasoning Criminal: Rational Choice Perspectives on Offending. New York, NY: Springer-Verlag (1986)

10. Kongmuang, C.: Modelling Crime: A Spatial Microsimulation Approach. PhD thesis, University of Leeds, UK (2006)

11. Bonabeau, E.: Agent-based modeling: Methods and techniques for simulating human systems. In: Proceedings of the National Academy of Sciences, 99, pp. 7280-7287 (2002)

12. Canessa E., Quezada A.: Modelamiento basado en agentes: un método de simulación computacional para la gestión de seguridad ciudadana. Universidad Adolfo Ibañez, Chile (2013)

13. Devia N.: Generación de datos de delincuencia vía simulación con modelos basados en agentes. Facultad de Ciencias Físicas y Matemáticas, Departamento de ingeniería industrial, Universidad de Chile, Santiago Chile (2012)

14. Amrutha, S.: Agent Based Simulation of Street Robbery. Department of computer science, Royal college of engineering and technology Thrissur, Kerala, India (2014)

15. Malleson, N.: Agent-Based Modelling and Crime in Leeds. School of Geography, University of Leeds (2007)

16. AnyLogic: Multimethod Simulation Software, http://www.anylogic.com/

17. AnyLogic: Application Areas, http://www.anylogic.com/application-areas 